\title{
The potential impact of periodontitis on the pathogenesis of Parkinson's disease and cognitive deficits
}

\author{
T.Y. Renn ${ }^{1}$, L.Y. Chen², H.-M. Chang ${ }^{3 *}$ \\ ${ }^{1}$ Graduate Institute of Medical Sciences, Taipei Medical University, Taipei, Taiwan \\ ${ }^{2}$ Department of Anatomy, Chung Shan Medical University, Taichung, Taiwan \\ ${ }^{3}$ Department of Anatomy and Cell Biology, Taipei Medical University, Taipei, Taiwan \\ *e-mail:taiwanzoo@gmail.com
}

Key words: periodontitis, systemic inflammation, neuroinflammation, Parkinson's disease

\begin{abstract}
Motivation and Aim: Previous studies have highlighted the importance of inflammatory reaction in the initiation and progression of neurodegenerative disorders. Periodontitis is one of the most prevalent chronic inflammatory diseases, which increased inflammatory burden that forms the basis for the proposed link to various systemic diseases such as cardiac diseases, diabetes, renal diseases, low birth weight as well as Alzheimer disease. However, although chronic inflammation is consistently associated with the pathophysiology of Parkinson's disease, so far, there is no direct evidence interlinking the detrimental effects of periodontitis on the pathogenesis of Parkinson's disease. In this regard, it is important to clarify the potential impact of periodontitis on the induction or development of Parkinson's disease.
\end{abstract}

Methods and Algorithms: Both in vitro and in vivo approaches were utilized to clarify the potential impact of periodontitis on the development of Parkinson's disease. For the in vitro study, both LUHMES cell and BV2 cell, representing the midbrain dopaminergic neuron and microglia, respectively, were exposed to periodontal pathogen-derived lipopolysaccharide (P.g. LPS) to demonstrate the cellular responses and the potential signaling pathway involved in the pathogenesis of Parkinson's disease induced by periodontitis. For the in vivo study, the pathological changes as well as the related neurochemical expressions in the substantia nigra and hippocampus were extensively examined by the use of two animal models: (1) directly infecting the periodontal tissue with periodontal pathogens, and (2) tightly encompassing the periodontal tissue with cotton threads.

Results: In vitro studies showed that although P.g. LPS did not affect the cell viability of both LUHMES and BV2 cells, it actually induced the oxidative stress and up-regulated the cytokine levels that may ultimately contribute to neuroinflammation. In vivo studies showed that decreased tyrosine hydroxylase, increased glial fibrillary acidic protein, and enhanced $\alpha$-synuclein immuno-expressions were all detected in the SN of rats subjecdted to periodontitis.

Conclusion: As periodontitis significantly depressed TH expression and induced the expression of $\alpha$-synuclein, one of the most important pathological features of Parkinson's disease, these evdiences thus support the close interaction between periodontitis and the development of Parkinson's disease.

Acknowledgements: Supported by the Ministry of Science and Technology (MOST 1062633-B038-002). 